\title{
Matrix Effect in Pesticide Analysis by Ultra Fast Liquid Chromatography Coupled to Mass Spectrometry
}

\author{
Diego A. Ahumada, ${ }^{*, a}$ Adriana M. Zamudio ${ }^{a}$ and Julio C. España ${ }^{b}$ \\ ${ }^{a}$ Laboratorio de Inocuidad Química, Centro de Bio-Sistemas, Facultad de Ciencias Naturales e Ingeniera, \\ Universidad de Bogotá Jorge Tadeo Lozano, Bogotá, Colombia \\ ${ }^{b}$ Departamento de Química, Facultad de Ciencias, Universidad Nacional de Colombia, \\ Bogotá, Colombia
}

\begin{abstract}
O presente trabalho descreve os resultados de um estudo de diferentes variáveis que influenciam o efeito matriz (EM). Para isso, optamos entre quatro diferentes solventes de extração e quatro métodos de limpeza, a fim de determinar qual é o método que produz um menor efeito matriz e uma maior resposta cromatográfica para os 18 compostos testados. Estudamos também a variação das respostas cromatográficas usando cinco tipos diferentes de frutas. Finalmente, foram avaliadas diferentes relações analito/matriz. Os resultados mostraram que as respostas significativamente melhores $(\mathrm{P}<0,05)$ foram obtidas usando a mistura de três diferentes absorventes, e o negro de carbono grafitizado ocasionou a maior supressão da ionização. Das cinco matrizes avaliadas, descobrimos que as uvas e extrato de tomates causaram maior efeito matriz, enquanto que a manga e maçã apresentaram os melhores sinais cromatográficos. Finalmente, encontramos uma correlação negativa entre a proporção de analito/matriz e a supressão da ionização.
\end{abstract}

In this work a study of several variables that affect the matrix effects (ME) was performed. The choice was given among four extraction solvents and four different clean-up methods which were compared in order to find the method that produces least ME and gives the highest response for 18 compounds. Variability of ME under identical chromatographic conditions for five fruits was also studied. Finally we evaluated different analyte/matrix ratios to assess the ME. The results showed that the significantly better responses $(\mathrm{P}<0.05)$ were achieved by using the mixture of three absorbents and the use of only Graphitized Carbon Black causes greater suppression of ionization. From the five matrices tested it was found that grapes and tomatoes extract induced a larger ME, meanwhile the use of apple and mango matrices gave responses more like matrix-free standards. Finally, a negative correlation between the suppression of ionization and the compound/ matrix ratio was found.

Keywords: pesticides, mass spectrometry, liquid chromatography, matrix effect

\section{Introduction}

At the early stage of the last decade, liquid chromatography (LC) coupled mass spectrometry has become of utmost importance in the field of environmental residues analyses. ${ }^{1}$ Mainly, this preference has emerged by the huge variety of physicochemical properties of these compounds. The great versatility in LC enables the determination of a larger kind of chemical moieties by such means. Regarding more recent technological developments, as quads, ion traps and other mass filters, mass spectrometry had acquired a high specificity and selectivity. This trend

*e-mail: diego.ahumadaf@utadeo.edu.co has led to an almost complete abolition of interferences at the target signal entry. Such advances had turned out in a marked tendency to the development of analytical techniques showing the least possible number of steps in sample processing and a minimal chromatographic retention, such as the QuEChERS method ${ }^{2}$ does in itself. However, in most cases due to the coelution of organic and inorganic compounds with the analyte ${ }^{3}$ and depending on the environment where the ionization takes place 4,5 either an enhancement or suppression effect of the signal is perceived. It is believed that changes in the ionization efficiency in presence of other compounds should be caused by non-volatile material and high surface activity compounds. ${ }^{6,7}$ 
The matrix effect (ME) in residue analyses is one of the main pitfalls at the time of the identification and quantification of contaminants, because it affects by far the reproducibility and accuracy of the method. Specifically, the matrix effect in LC-MS refers to the alteration in the performance of the ionization process by the presence of co-eluants. ${ }^{4}$ According to Antignac et al. ${ }^{8}$ the suppression mechanism assumes several sceneries. First of all, the ionization efficiency is decreased because the solvent evaporation is hindered by an increase of the viscosity and the surface tension. Secondly, macromolecules and non-volatile material co-precipitate with analytes. Finally, a suppression path already in gas phase includes a neutralization reaction due to the increase of the basicity.

At the laboratory these effects are generally coped using matrix-matched calibration curves, nonetheless Kruve et al. ${ }^{9}$ has proved the intense dependence of the type of matrix on this effect even into varieties of the same product. There is a pool of alternatives to overcome this phenomenon either by matrix effect minimisation or compensation, ${ }^{10}$ for instance making use of a labelled internal standard, ${ }^{11}$ changing the interface conditions (i.e., nanospray ${ }^{3}$ ), improving the chromatographic separation, ${ }^{8}$ cleaning the extract during the sample processing even more ${ }^{12}$ or holding the standard addition method.

Therefore, for all these reasons the evaluation of the matrix effect takes place in the present work due to its undeniable importance. In this study we made use of the post-extraction addition technique, with the aim of studying different variables that affect the suppression process determining the ME, such as the type of matrix employed, the concentration effects, the clean-up method, the extraction solvent and the amount of matrix.

\section{Experimental}

\section{Reference materials, reagents and solutions}

Pesticide reference standards, all $>95 \%$ purity, were obtained from Dr. Ehrenstorfer GmbH (Augsburg, Germany) and Chemservice (West Chester, PA, USA). Stocks were prepared in a concentration around $500 \mu \mathrm{g} \mathrm{mL}^{-1}$, using either, acetonitrile or methanol as solvent, and were stored in amber glassware under appropriate conditions such as $-20{ }^{\circ} \mathrm{C}$, exclusion of moisture and light. The mixture of pesticides was made in methanol by measuring different volumes of each stock in order to obtain a concentration range between $0.64 \mu \mathrm{g} \mathrm{mL}^{-1}$ to $9.95 \mu \mathrm{g} \mathrm{mL}^{-1}$. In Table 1, the common names and their concentration levels made from appropriate dilutions of the mixture are given. All the solvents used were HPLC grade J.T. Baker (Phillipsburg, NJ, USA). The QuEChERS Restek Q-Sep ${ }^{\text {TM }}$ salt kits were used in the extraction process and the Restek dSPE Q-Sep ${ }^{\mathrm{TM}}$ adsorbent kits were employed in the clean-up procedure.

Table 1. Compounds and concentration levels used along the study (L1 = Level 1; L2 = Level 2; L3 = Level 3; L4 and L5 = Level 5)

\begin{tabular}{|c|c|c|c|c|c|c|}
\hline \multirow[t]{2}{*}{ Compound } & \multirow[t]{2}{*}{ Classification } & \multicolumn{5}{|c|}{ Concentration / $\left(\mu \mathrm{g} \mathrm{mL}^{-1}\right)$} \\
\hline & & L1 & L2 & L3 & L4 & L5 \\
\hline Triphenylphosphate ${ }^{\mathrm{a}}$ & Organophosphorus & 0.03 & 0.04 & 0.06 & 0.07 & 0.09 \\
\hline Azoxystrobin & Strobylurin & 0.05 & 0.08 & 0.10 & 0.13 & 0.15 \\
\hline Imidacloprid & Pyridilmethylamine & 0.20 & 0.30 & 0.40 & 0.50 & 0.60 \\
\hline Dimethoate & Organophosphorus & 0.09 & 0.13 & 0.17 & 0.22 & 0.26 \\
\hline Azinphos-methyl & Organophosphorus & 0.13 & 0.20 & 0.27 & 0.33 & 0.40 \\
\hline Diazinon & Organophosphorus & 0.01 & 0.02 & 0.03 & 0.04 & 0.05 \\
\hline Imazalil & Conazole & 0.05 & 0.08 & 0.10 & 0.13 & 0.15 \\
\hline Acephate & Organophosphorus & 0.14 & 0.22 & 0.29 & 0.36 & 0.43 \\
\hline Pyrazophos & Organophosphorus & 0.05 & 0.07 & 0.10 & 0.12 & 0.15 \\
\hline Monocrotophos & Organophosphorus & 0.02 & 0.03 & 0.04 & 0.05 & 0.06 \\
\hline Carbofuran & Carbamate & 0.03 & 0.05 & 0.07 & 0.09 & 0.10 \\
\hline Isophenphos & Organophosphorus & 0.01 & 0.02 & 0.03 & 0.04 & 0.05 \\
\hline Tebuconazole & Triazole & 0.03 & 0.04 & 0.05 & 0.06 & 0.08 \\
\hline Malathion & Organophosphorous & 0.08 & 0.12 & 0.16 & 0.20 & 0.24 \\
\hline Spinosad & spinosyn & 0.15 & 0.22 & 0.29 & 0.37 & 0.44 \\
\hline Difenoconazole & Triazole & 0.11 & 0.17 & 0.23 & 0.28 & 0.34 \\
\hline Metalaxyl & Anilide & 0.07 & 0.10 & 0.13 & 0.17 & 0.20 \\
\hline Carbendazim & Becimidazolil carbamate & 0.12 & 0.19 & 0.25 & 0.31 & 0.37 \\
\hline
\end{tabular}

aTriphenylphospahte (TPP) does not belong to the pesticides group, it is the internal standard most often used in pesticide analyses. 


\section{Equipment}

The chromatographic analyses were performed in an ultra-high speed liquid chromatograph Shimadzu Prominence $^{\mathrm{TM}}$ coupled to a LCMS-2020 mass selective detector (Maryland, CA, USA). An ABN2ZE Peak Scientific (Billerica, USA) nitrogen generator was employed to provide the dryer stream in the ESI source. The chromatograph was equipped with a SIL20A UFLC 7673 Shimadzu (Maryland, CA, USA) automatic sampler, a binary high pressure pump, online degasification system and an oven to control the column temperature. The acquisition, control and data processing were performed using the Lab Solutions version 3.5 software.

\section{Chromatographic conditions}

The analyses were performed with a Shim-pack C18 column (60 $\mathrm{mm} \times 2 \mathrm{~mm}$ i.d., $2.1 \mu \mathrm{m}$ particle size). A linear gradient mode consisting of $0.1 \%$ formic acid $(\mathrm{m} / \mathrm{v})$ in Milli-Q grade water (A) and acetonitrile (B) were used to perform the separation. The programme used expressed as B percentage was: first $0 \%$ at $0.2 \mathrm{~min}$, afterwards a raising to $40 \%$ at $1 \mathrm{~min}$ and then again to $100 \%$ in the next $7 \mathrm{~min}$, and finally a hold time by $1 \mathrm{~min}$. Thereafter, the initial condition was restored in $2 \mathrm{~min}$, where it was held by $6 \mathrm{~min}$ to reach equilibrium. The total analysis time was $9 \mathrm{~min}$. The flow rate of the mobile phase was $0.3 \mathrm{~mL} \mathrm{~min}^{-1}$ at column temperature $40{ }^{\circ} \mathrm{C}$. An aliquot of $5 \mu \mathrm{L}$ of the extracts was injected into UFLC system.

\section{Interface conditions and mass spectrometer}

A built-in DUIS (ESI, APCI) interface was used operating in ESI mode, a drying gas flow of $15 \mathrm{~L} \mathrm{~min}^{-1}$ and a nebulizer gas flow of $1.5 \mathrm{~L} \mathrm{~min}^{-1}$. The temperatures of the heating block and the desolvation line were $200{ }^{\circ} \mathrm{C}$ and $250{ }^{\circ} \mathrm{C}$, respectively. The analysis was carried out in both positive and negative modes; the applied voltage at the capillary was $4500 \mathrm{~V}$ and $-4500 \mathrm{~V}$, respectively. All the analyses were performed in single ion monitoring mode; Table 2 shows the selected ions and the ionization mode for each compound.

\section{Extraction study}

Pesticide-free black grapes, mangoes, tomatoes, lulos and apples were obtained at the local market. Besides, they were carefully washed with generous amounts of water and any strange material on the surface was chucked. Finally, the samples were assessed by LC-MS to ensure they were
Table 2. Identification parameters of targeted pesticides obtained by UFLC/ESI-MS. Retention time (RT), target ion (T) and qualifiers (Q1 and Q2)

\begin{tabular}{|c|c|c|c|c|}
\hline Compound & $\mathrm{RT} / \mathrm{min}$ & $\mathrm{TI}(m / z)$ & $\mathrm{Q} 1(\mathrm{~m} / \mathrm{z})$ & $\mathrm{Q} 2(\mathrm{~m} / \mathrm{z})$ \\
\hline \multicolumn{5}{|c|}{ Positive ionization mode } \\
\hline TPP & 7.00 & 327 & 368 & \\
\hline Azoxystrobin & 5.95 & 404 & 405 & 372 \\
\hline Dimethoate & 3.70 & 230 & 199 & 171 \\
\hline Azinphos-methyl & 7.10 & 346 & 318 & 289 \\
\hline Diazinon & 7.00 & 305 & 306 & \\
\hline Imazalil & 4.70 & 297 & 299 & \\
\hline Acephate & 2.10 & 389 & 184 & 241 \\
\hline Pyrazophos & 7.15 & 374 & 396 & 415 \\
\hline Monocrotophos & 3.85 & 224 & 242 & 241 \\
\hline Carbofuran & 4.20 & 222 & 165 & \\
\hline Isophenphos & 7.05 & 346 & 287 & 245 \\
\hline Malathion & 5.84 & 331 & 348 & \\
\hline Spinosad A & 6.35 & 733 & 736 & \\
\hline Spinosad D & 6.55 & 747 & 749 & \\
\hline Difenoconazole & 7.00 & 406 & 408 & \\
\hline Metalaxyl & 4.45 & 280 & 281 & \\
\hline Carbendazim & 3.50 & 192 & 233 & \\
\hline \multicolumn{5}{|c|}{ Positive/negative ionization mode } \\
\hline Imidacloprid & 4.05 & 256 & 297 & -254 \\
\hline Tebuconazole & 4.80 & 308 & 310 & -352 \\
\hline
\end{tabular}

pesticide-free material. The QuEChERS method $^{2}$ was carried out in order to obtain pesticide-free blank extracts by the procedure explained next. In a centrifuge tube, $15 \mathrm{~g}$ of previous homogenised sample were weighted, $15 \mathrm{~mL}$ of solvent (acetonitrile) were poured into and then it was manually shaken by $1 \mathrm{~min}$. In particular, the extraction solvent study using acetonitrile, ethyl acetate, methanol and a mixture of 50:50 acetonitrile and methanol was performed only over tomatoes. Thereafter, $6 \mathrm{~g}$ of anhydrous $\mathrm{MgSO}_{4}$ and $1 \mathrm{~g}$ of sodium acetate were added, it was shaken then again. The tube was centrifuged to $4500 \mathrm{rpm}$ for $5 \mathrm{~min}$ and $10 \mathrm{~mL}$ of the supernatant (solution A) were measured using a pipette and then transferred to a $15 \mathrm{~mL}$ centrifuge tube. In the case of the clean-up procedure, $25 \mathrm{mg}$ of PSA (primary/ secondary amine) and $150 \mathrm{mg}$ of anhydrous $\mathrm{MgSO}_{4}$ were added for each extract millilitre. Afterwards it was shaken by $30 \mathrm{~s}$ and centrifuged by $2 \mathrm{~min}$ at $5000 \mathrm{rpm}$. Finally, the supernatant was filtered through a $0.22 \mu \mathrm{m}$ PTFE filter (solution B). Pesticides solutions in neat solvent and matrix extract (solution B) were prepared in order to evaluate the type of matrix. For that purpose, $40 \mu \mathrm{L}$ of the pesticides mixture were placed into a $1 \mathrm{~mL}$ volumetric flask and the volume was completed either with solution B or neat solvent (corresponding to level 2 in Table 1). The method was previously optimized and validated. ${ }^{13}$ 


\section{Clean-up study}

Several adsorbents and combinations of them were assessed to do the clean-up procedure. For this purpose, $2 \mathrm{~mL}$ of the solution B were measured, transferred to a $15 \mathrm{~mL}$ centrifuge tube and the quantities of adsorbent in Table 3 were carefully added.

Table 3. Influenced of amount and type of absorbent on the matrix effects

\begin{tabular}{lccc}
\hline Method & $\mathrm{PSA}^{\mathrm{a}} / \mathrm{mg}$ & $\mathrm{GCB}^{\mathrm{b}} / \mathrm{mg}$ & $\mathrm{C}_{18} / \mathrm{mg}$ \\
\hline 1 & 50 & 0 & 0 \\
2 & 100 & 0 & 0 \\
3 & 0 & 50 & 0 \\
4 & 50 & 50 & 0 \\
5 & 50 & 50 & 50 \\
\hline
\end{tabular}

aPrimary-secondary amine (PSA); ' Graphitized Carbon Black (GCB); 'Octadecylsilane $\left(\mathrm{C}_{18}\right)$.

Finally, all the results in this study were performed by triplicate and using complete randomised designs. The statistical analyses were made at a significance level of $5 \%$ making use of the SPSS ${ }^{\mathrm{TM}}$ statistical package software.

\section{Amount of matrix study}

In order to study how the amount of matrix injected with the pesticides affects the phenomenon, different values of matrix concentration were tested $\left(0.5 \mathrm{~g} \mathrm{~mL}^{-1}, 1.0 \mathrm{~g} \mathrm{~mL}^{-1}\right.$ and $\left.2.0 \mathrm{~g} \mathrm{~mL}^{-1}\right)$. A volume of $40 \mu \mathrm{L}$ of the pesticides mixture and $500 \mu \mathrm{L}$ of matrix extract were added to a $1 \mathrm{~mL}$ volumetric flask, acetonitrile was used to complete to volume to make solutions at a level of matrix concentration of $0.5 \mathrm{~g} \mathrm{~mL}^{-1}$. On the other hand, with the aim of preparing solutions with a matrix concentration of $2 \mathrm{~g} \mathrm{~mL}^{-1}$, a concentration step of the solution $\mathrm{B}$ was needed, so that $2 \mathrm{~mL}$ of this extract were left in a $2 \mathrm{~mL}$ PTFE tube and it was placed into a vacuum speed concentrator, where the volume was reduced to $c a$. $25 \%$ of its initial value. After, this extract was quantitatively transferred into a $1 \mathrm{~mL}$ volumetric flask, $40 \mu \mathrm{L}$ of the pesticides mixture were added and finally, acetonitrile was employed to complete to volume.

\section{Results and Discussion}

\section{Study of the extraction solvent}

It is known that extraction solvent choice is relevant in determination of analytes, even more in residue analysis where the effectiveness of the extraction capacity is a significant issue of concern. Despite of the solvent ability, mainly in drawing the compounds of interest out to the probe, in this experiment we want to explore the possibility of any effects over the capability of the mass detection system for the same level of pesticide concentration (second level of the trial concentrations in Table 1) caused by matrix co-extracted components present in tomato. In order to determine to what extent the solvent co-extractives effectively affect the response, the results of this completely randomized design (CRD) were analysed using an analysis of variance (ANOVA). The performance of this analysis was only done afterwards a carefully and systematic checking of the assumptions hereafter: first of all, that the experimental error is an independent random variable due to a randomly choice of fruits, secondly that it follows a normal distribution proved by the Shapiro-wilk test, thirdly that its mean is equal to zero reckoned by least squares method and finally that the design has homogenous variances tested by Levene's test. Any case of heterogeneity of variances led to the discarding of the data, while in the case of a moderate departure from normality, the results were conserved because it does not necessary imply a serious violation of the assumptions and also because Levene's test is rugged enough in experiments where small samples are employed. The ANOVA demonstrates significant differences $(\mathrm{P}<0.05)$ between the responses for all the pesticides of the study aside from acephate. This is a fact which pointed out the importance of the solvent choice. This issue strongly affects the detection of most compounds. With the purpose of contrast the pesticide area means using each solvent, a Tukey's test was performed (Figure 1). To develop this test the means are ordered in letters increasingly and joined together in a group only if no least significant difference is reckoned at a $95 \%$ level of confidence. The figure 1 shows that near the $90 \%$ of pesticides extracted with acetonitrile scored the group with the highest response. As well, an important amount

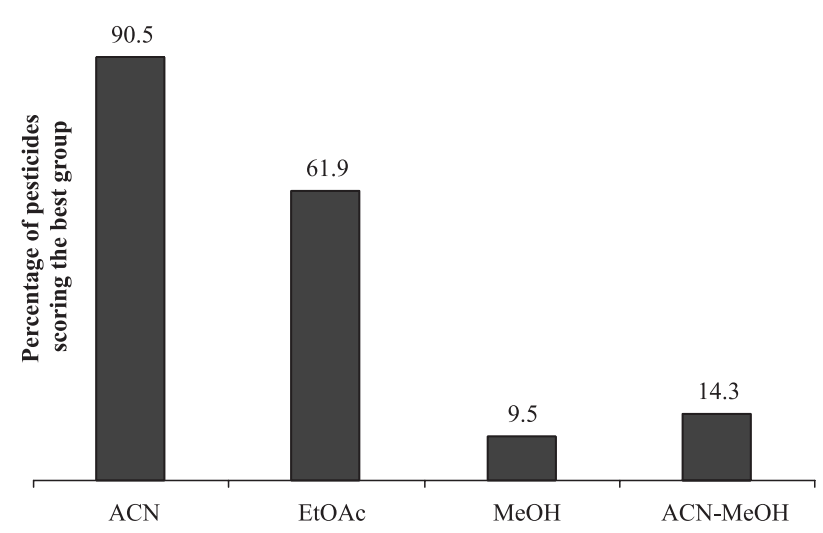

Figure 1. Fraction of compounds with the highest chromatographic response, according to the Tukey's test $(\mathrm{P}<0.05)$ for the comparison of extraction solvents. 
of pesticides reached the best position using ethyl acetate as solvent. On the other hand, when using methanol as extraction solvent only nearly $10 \%$ of the pesticides are classified in the group with highest response. In general, for this solvent it was found that nearly $50 \%$ of the compounds present a very low response. The Tukey's test, showed that the worst response all over the solvents was reached with ethyl acetate only for a minority of pesticides.

It is evident that the highest responses are reached using acetonitrile, this outcome would be explained because there is no presence of matrix co-extractive components which compete against the analytes inside the detection system or probably those effectively extracted are, in nature, unable to interfere with them over the ionization process into the ESI interface. The importance of the choice of the best solvent is not only restricted to the best recovery as is usually considered, though it is always desirable. Another concern is found in the detection system. Sometimes, an increase in recovery of polar compounds is expected using a combination of solvents (in this case using a 50:50 acetonitrile:methanol mixture). Nevertheless, it is clear that in this case a lower detectability of the same concentration level is reached since methanol would extract compounds from the matrix that possibly drive to ionization suppression of the pesticides. In the further experiments, it is used only acetonitrile to prepare blank extracts to avoid ionization suppression from this source.

\section{Study of clean-up methods}

Another topic developed in this study has to do with the clean-up adsorbents employed in QuEChERS methodology. As the extractive power of acetonitrile regarding to the rest of solvents tested, does not affect the response of the pesticides in tomato extracts, a number of different adsorbents commonly used in pesticide analyses on fruits and vegetables were tried to give the lower signal suppression. We made use of GBC, PSA and $\mathrm{C} 18$ combinations in Table 3 since they are the more often used adsorbents in practice. A CRD was performed and an ANOVA demonstrates that there were significant differences $(\mathrm{P}<0.05)$ by employing these adsorbents for all the pesticides tested. Using the Tukey's test the area responses were organised in groups in the same way of the previous explanation. Only the best one is shown in Figure 2.

High dependence of both the presence and the amount of PSA could be noticed at the Figure 2 while the worst responses for all the compounds was achieved using only GCB. Additionally, combinations of adsorbents with PSA and the larger its amount, the more analytes belong

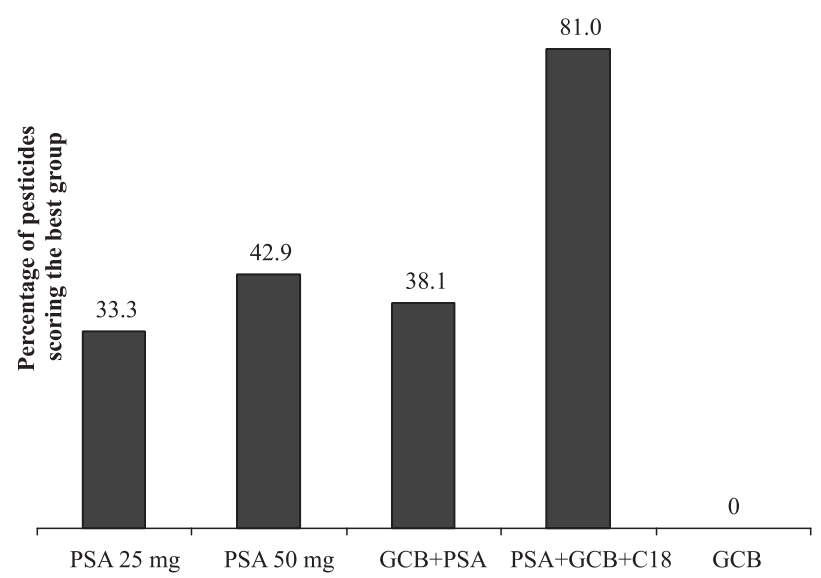

Figure 2. Fraction of compounds with the highest chromatographic response, according to the Tukey's test $(\mathrm{P}<0.05)$ for the study of clean-up adsorbents; $\mathrm{PSA}=$ primary-secondary amine; $\mathrm{GCB}=$ graphitized carbon black and octadecylsilane (C18).

to the best group where higher responses were obtained. Thereby, a combination of adsorbents in adequate amounts is recommended. In the forthcoming studies, the clean-up was performed by using PSA as the adsorbent of choice.

On other hand, the Figure 2 shows that the use of only GCB causes greater suppression of ionization than the others sorbents. These results would be explained because the Graphitized Carbon Black show strong binding affinity for hydrophobic molecules; thereby, this adsorbent can remove some compounds such as pigments, sterols, and nonpolar interferences. However molecules like sugars and fatty acids, e.g. carotenoids, are not removed from the matrix extracts. According above for tomato matrix this clean-up process is not enough effective and causes an increase at the suppression of the chromatographic signals.

\section{Matrix effect and amount of matrix}

The estimation of matrix effects over different amounts of matrix was carried out at three scales using the level 2 of the calibration curve. Significant differences $(\mathrm{P}<0.05)$ of the correspondent CRD were found by using the Dunnett's test between each one of the five different matrices compared always against the matrix-free standard. The results show both suppression and enhancement of the signal, but the latter was present for only two pesticides, first for monocrotophos at a matrix amount of $1 \mathrm{~g} \mathrm{~mL}^{-1}$ and $2 \mathrm{~g} \mathrm{~mL}^{-1}$, and it also for the signals of spinosysn A and $\mathrm{D}$ at all levels. The odd behaviour of monochrotophos is unexpected and there are no experimental evidences that could explain this event. In the evaluation of the blank extracts chromatograms, it was found no interference at least for the target ion mass range in the time window of spinosyns A and D. Since the results for both compounds 
shows a consistent enhancement of the signal, the occurrence of an unusual matrix enhancement in this technique leads to think there is a completely different mechanism present in the ionization of this large-sized molecule. It could be possible that molecules alike show a fairly higher surface activity because of their peculiar structure, being readily charged in the offspring droplets. It is very relevant to point out that ESI responses of analytes can vary significantly due to the sort of features that the structure meets. Cech et al. ${ }^{14}$ had considered for their study that analytes with high surface activity can carry a greater fraction of excess charge and thereby they produce higher ESI responses. Accordingly, higher responses in every matrix extract are achieved only with spinosyns, which have some nonploar regions producing a strong affinity for the surface, this exclusively enables to this kind of pesticides to compete with endogenous matrix compounds in the droplets. From the molecular structure of spinosyns A and D in Figure 3 it is possible to observe that there is a large amount of oxygenated groups and reaction targets, ranging from carbonyl functional groups to reactive double bonds which may be prone of a feasible ionization. Moreover, these voluminous structures could compete and even more they should increase the possibilities of ionization when matrix components are all around, improving in such a way its responsiveness. The target ion used in the determination of spinosad was the protonated spinosyns A and D. There is not a reliable fragmentation pattern of any spinosyns, and therefore only this high mass-charge ratio could be used to identify them. Apparently, the protonated adduct of this molecule take advantage of the ionization chamber environment being more stable and a more frequently ion at the ESI interface than others adducts, increasing readily the formation of positively charged species. When co-elutant matrix components are present the environment is enriched of a combination of low and high energy molecules that triggers the stability of this molecule transferring charge
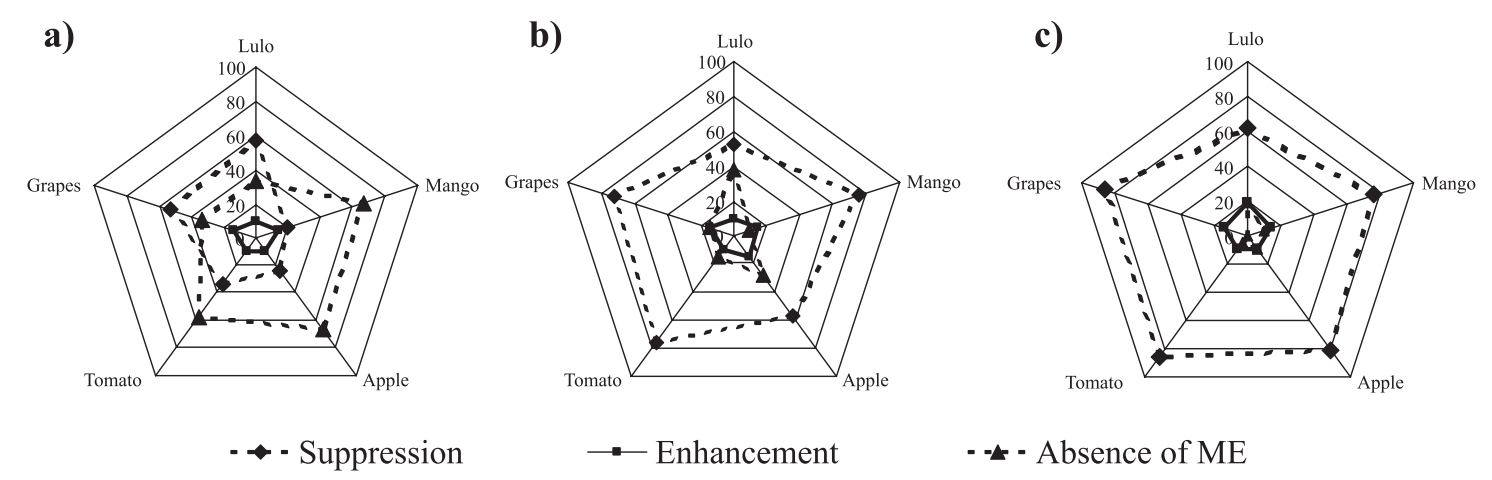

Figure 4. Percentage of the compounds showing a possibly matrix effect in lulo, mango, apple, tomato and grapes extracts. (a) Extracts at $0.5 \mathrm{~g} \mathrm{~mL} \mathrm{~m}^{-1}$ of

matrix, (b) extracts at $1 \mathrm{~g} \mathrm{~mL}^{-1}$ of matrix and (c) extracts at $2 \mathrm{~g} \mathrm{~mL}^{-1}$ of matrix. from the manifold short fragments to the voluminous moiety. In addition, the gas-phase proton-transfer reactions may add up to the effectiveness of ionisation, meanwhile this sort of phenomena in general depletes the number of charged analyte by an inversion of the basicity in the gas phase. This interactions, if it really take place right afterwards that the analyte is released from solution, may lead to a higher gas-phase affinity that seems to be in particular a profitable for this kind of molecules. This increase of ionization efficiency leads to a gain in sensibility achieving the lowest limits of detection among other pesticides determined by the same mode of ionisation as some authors ${ }^{15}$ recently had reported.

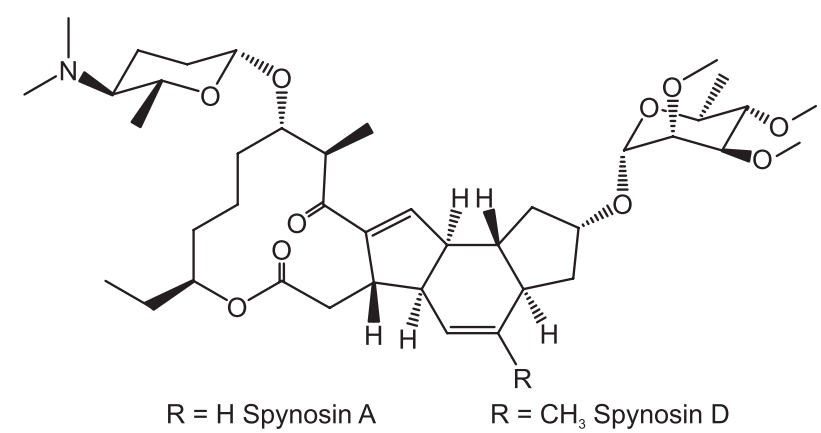

Figure 3. Molecular structures of spinosad (Spynosin A and Spynosin D).

An account of the percentage of pesticides showing any matrix effects has been plotted in Figure 4 for all the matrices studied. It can be seen, that most compounds exhibit rather suppression than an enhancement effect. Besides, it is noticed that the enhancement effect is almost constant between the amounts of matrix in the extracts. The number of compounds showing ionisation suppression in extract of lulo seems to remain invariable for all levels.

Careful study of the absence of matrix effect considered as the lack of significant differences $(\mathrm{P}<0.05)$ between the signal of the pesticide in solvent and its response acquired in matrix extracts of each fruit or vegetable, drive us at the conclusion of a highly dependence of the matrix effect with 
the compound/matrix ratio. This deduction arose from the tendency of the increasing number of pesticides undergoing suppression of the signal in all matrices and at the same time a remarkable decrease of the unaffected compounds at higher amount of matrix levels. Compounds such as acephate, monocrotophos, spinosyn D, imazalyl, TPP and diazinon were left out of the account only in certain levels because their variances were found not to be homogenous. It is important to highlight that in most cases no significant differences were found for TPP, compound often used as the internal standard, nonetheless it was never used for correction proposes, this time it was only about its evaluation.

\section{Matrix effect and pesticide concentration}

Despite of the fact that ME is usually assessed by means such as two-tailed paired test or by regression analysis, drawbacks arising from the lack of concordance between the results of these classical statistical techniques with the features directly observed in the chromatograms, prompt us to estimate the presence of the ME using a straight comparison with the matrix-free standards response in order to determine a possibly ME at a certain level of confidence.

Assessment of significant differences $(\mathrm{P}<0.05)$ of the responses between matrix-free standards as reference and extracts of tomato and apple for the whole set of pesticides by Dunnett's test was performed over the range of calibration levels showed in Table 1. As the Figure 5 shows, a noteworthy number of compounds that presents signal suppression in tomato remains in a consistent value near 18 compounds all over the levels. Unlike tomato, apple extract shows at low levels a large number of compounds that resembles matrix-free standard responses, but it also shows an increasingly number of pesticides undergoing suppression as the concentration arises from the lowest to the highest level, reaching at level five of concentration the same amount of compounds suppressed in tomato. Otherwise, the enhancement effect remains restricted to only spinosad in extract of tomatoes because in apple at the lowest level it has the higher area value but then decreases with the concentration.

\section{Type of matrix influence on pesticides responses}

The response of pesticides may vary in a fair extent depending on the sort of matrix in which the same analyte/ matrix ratio is measured. Similarly to the study over the levels of amount of matrix, a comparison between matrices in a CRD using a factorial structure was accomplished. In Figure 4 it can be seen that the number of pesticides showing significant differences between matrix-free injections and in lulo and grapes extracts behaves similarly over the compound/ matrix ratios regarding to the others. Indeed, it is also possible to recognise a slight difference of the number of pesticides undergoing ME in apple rather than in tomato and mango extracts. With the aim of finding out differences between the nature of matrices, and on the basis of the previously observed trends, a series of contrasts has been made. In a set of four hypotheses, significant differences $(\mathrm{P}<0.05)$ were found for most of pesticides where each postulate is directly related with a proper orthogonal contrast which serves as proof of significance as per the following arrays:

The results for all the comparisons are summarised in Figure 6 the total of pesticides showing statistical differences for every hypothesis was reckoned as percentage, leaving out the mentioned compounds that exhibit heterogeneity of variances. Therefore, from the first hypothesis it is evident that only at the lowest analyte/matrix ratio, above the $90 \%$
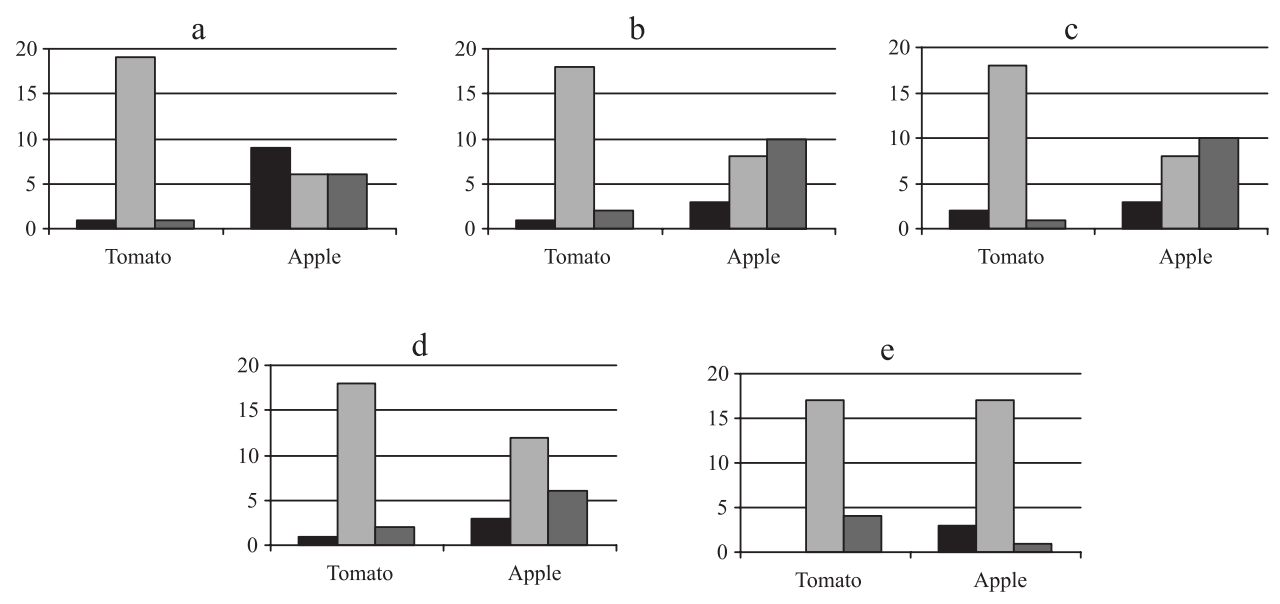

Enhancement

$\square$ Suppression

$\square$ Absence of ME

Figure 5. Effect of concentration levels in the pesticide signal solvent, tomatoes and apple extracts. Graphs (a), (b), (c), (d) and (e) correspond to levels 1, $2,3,4$, and 5 of the calibration curve respectively in selected matrices. 
of the pesticides in the extracts that hold the highest ME such as lulo and grapes showed significant differences when comparing them with the rest of matrices. This trend suggests that large suppression in reached for most pesticides when high amount of matrix is used. On the contrary, near the $60 \%$ of pesticides at the lowest level of matrix reveal the previously noted dissimilarity in responses between lulo (that remains almost constant in Figure 4) and the mean of extract of apple and tomatoes. In addition, to put into the test the clusters we made, it was compared the matrices within each group. The outcomes disclose that only around $30 \%$ of the pesticides exhibit significant differences for the both contrasts, firstly between extracts of tomatoes and grapes $\left(\mathrm{H}_{\mathrm{oj}}\right)$ and secondly between mangoes and apple extracts $\left(\mathrm{H}_{\mathrm{o4}}\right)$. Nevertheless, it is noticeable that for the lowest amount of matrix there is no statistical difference. These results match with those in Figure 4 used to establish the clustering conditions of the comparison between matrices.

Table 4. Hypotheses made for comparing type of matrices

\begin{tabular}{l}
\hline $\mathrm{H}_{01} \quad \begin{array}{l}\text { The mean of the pesticide area in extracts of tomatoes and grapes is } \\
\text { equivalent to the mean of the pesticide area in extracts of mangoes, } \\
\text { apple and lulo. }\end{array}$ \\
$\mathrm{H}_{02} \quad \begin{array}{l}\text { The mean of the pesticide area in extract of lulo is equivalent to } \\
\text { the mean of the pesticide area in extracts of apple and mangoes. }\end{array}$ \\
$\mathrm{H}_{03} \quad \begin{array}{l}\text { The mean of the pesticide area in extract of tomatoes is equivalent } \\
\text { to the mean of the pesticide area in extract of grapes. }\end{array}$ \\
$\mathrm{H}_{04} \quad \begin{array}{l}\text { The mean of the pesticide area in extract of mangoes is equivalent } \\
\text { to the mean of the pesticide area in extract of apple. }\end{array}$ \\
\hline
\end{tabular}

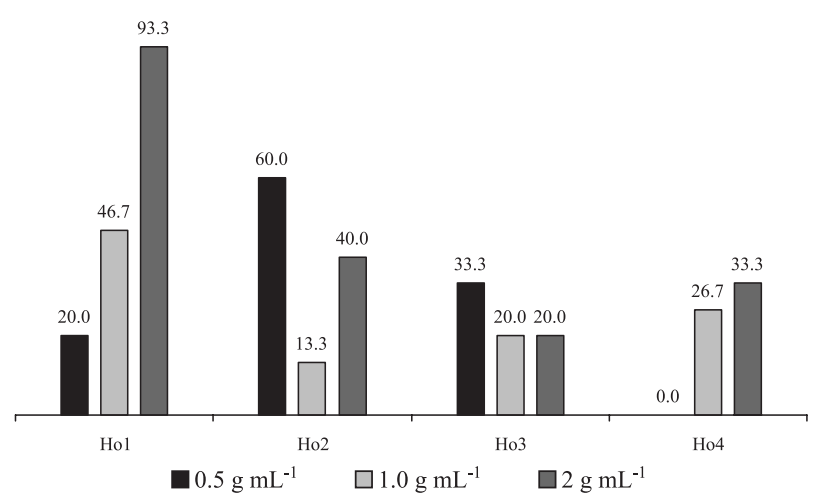

Figure 6. Percentage of pesticides showing significance in each contrast sorted by amount of matrix. $\mathrm{H}_{\mathrm{o} 1}, \mathrm{H}_{02}, \mathrm{H}_{03}$ and $\mathrm{H}_{\mathrm{o} 4}$ correspond to the hypotheses presented in Table 4 .

\section{Compound-matrix ratio influence on pesticides responses}

The effect of the amount of matrix in each item was studied. Three levels of amount of matrix were tested $\left(0.5 \mathrm{~g} \mathrm{~mL}^{-1}, 1 \mathrm{~g} \mathrm{~mL}^{-1}\right.$ and $\left.2 \mathrm{~g} \mathrm{~mL}^{-1}\right)$ at the second level of the pesticide calibration curve in a CRD for each matrix. Mainly, the previous suspicion of the close relation of amount of matrix with the suppression phenomenon for most of pesticides is observed in Figure 7 Results of percentage of pesticides scoring the highest group with significant differences from a Tukey's test $(\mathrm{P}<0.05)$ are presented as it was done before. In order to evaluate the differences between matrices it is enough to look at the lowest amount of matrix which in all matrices except in lulo, scored by far the higher response. Therefore, it was found for most compounds that the lower the compound/matrix ratio, the more ionization suppression the compound will undergo. In fact, from these experiments it is possible to recognise a negative correlation between the suppression of ionization and the compound/ matrix ratio. The other amounts of matrix tested were spread in the rest of groups. Nonetheless, despite that the lulo extract shows the highest values of area response at the $2 \mathrm{~g} \mathrm{~mL}^{-1}$ of amount of matrix, it is noticeable that the trend of the rest is completely opposite as expected.

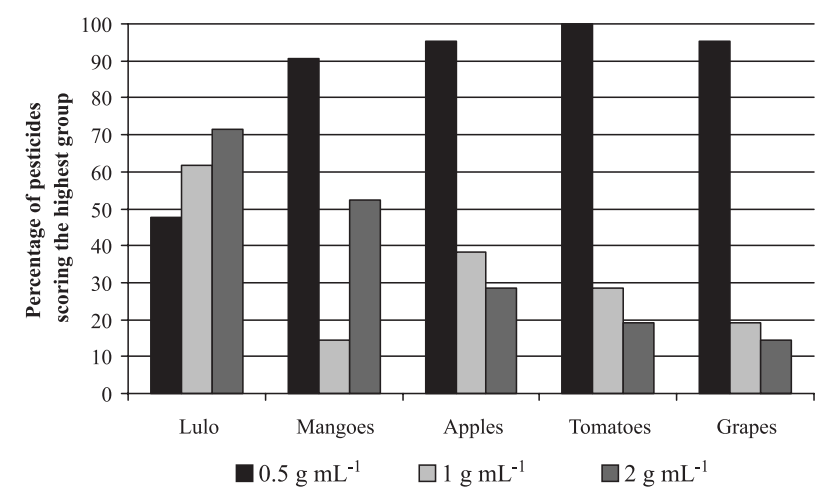

Figure 7. Effect of the amount of matrix in extracts. In the figure: (a) lulo, (b) mangoes, (c) apple, (d) tomatoes and (e) grapes.

Ionisation mode comparison

The values of ME calculated as the ratio between areas of the pesticide in matrix and neat solvent are showed in Figure 8 for the pesticides imidacloprid and tebuconazole in both positive and negative acquisition modes at level 2 of concentration and an amount of matrix of $1 \mathrm{~g} \mathrm{~mL}^{-1}$. As it is presented above, tomato and grapes show the lowest values of ME, indicating ionization suppression on the mainstream. In the case of imidacloprid, values very close to the $100 \%$ can be seen in negative operation mode at least when using this amount of matrix. The results of the positive mode, unlike the negative operation mode, show for all matrices a spread in a range from $120 \%$ to $80 \%$.

Excluding the results of imidacloprid, both ionization modes of tebuconazole are in appearance very similar, scattered around $80 \%$ for all matrices. The mechanism of ionization in the operational conditions of these compounds remains unknown. Some authors ${ }^{16}$ adduce that only successful negative ionization is reached when using stable anion precursors such as halogenated solvents, because 


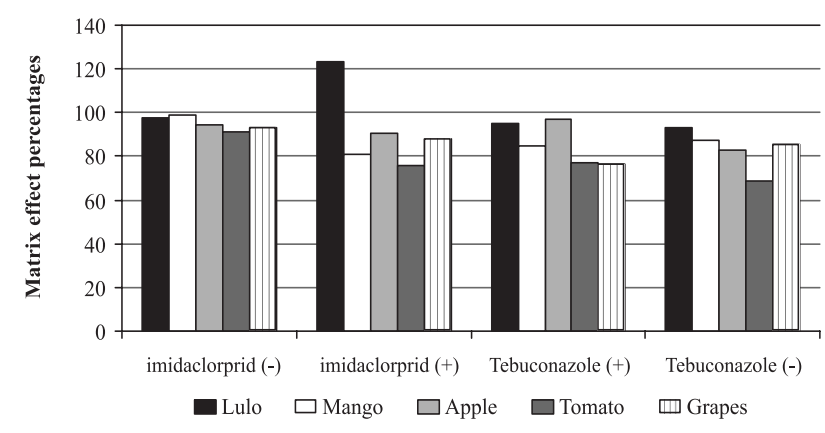

Figure 8. Matrix effect percentages for pesticides detected by both positive and negative modes.

of in attempt of improving negative ionisation by making use of basic aqueous solutions only poorer responses were obtained, however here appears that poor sensibility in negative mode affects the same way to both matrix-free standards and injected extracts.

\section{Conclusions}

A study of several variables which affect the response of a group of 18 compounds (including the internal standard) was carried out. The results obtained show that the extraction solvent and the clean-up method, using acetonitrile and the mix of the three absorbents, led to the best results in sensitivity terms showing the lowest signal suppression. Good precision was achieved for most compounds and assumptions of analyses of variance hardly ever failed. The study of ME demonstrates a strong dependence of the amount of matrix on injected extracts for all matrices. Broadly, the assessment of TPP, shows that no consistent ME was found. On the other hand, the concentration level shows a high influence on the ME of apple, unlike of tomato extracts which always have a large number of compounds featuring this effect independently from the level of concentration. There was only an issue that behaves in an absolutely seldom way. Spinosad, that mostly and constantly undergoes enhancement rather than suppression. This fact is explained by clear differences based in its noticeable larger and particular molecular structure. A comparison of the signal suppression between matrices was performed. Tomatoes and grapes were the matrices that show the most elevated ionization suppression from the set of study. Besides, the pesticides were more strongly affected by the increase of the amount of matrix in these extracts, leading to a negative correlation with the analyte/matrix ratio. The versatility of the equipment used allows evaluating ME of compounds in both positive and negative modes, showing that imidacloprid have some differences rather than tebuconazole. In practice, the positive ionization stays as the mode of choice for most compounds due to its superior sensibility. Nevertheless, further studies are required to completely characterize and explain the differences between matrices and extreme behaviours such as the high enhancement of spinosad or the eminent suppression triggered by the tomatoes extract. These possibilities include the exploration of the variability between other varieties of tomatoes and grapes using a larger set of pesticides.

\section{Acknowledgments}

The Authors are grateful to the Bio-Sistemas center and the funds from the Flemish Interuniversity Council (VLIR) through the project "Multidisciplinary assessment of efficiency and sustainability of smallholder-based tomato production systems in Colombia, with a roadmap for change" (code: ZEIN2009PR364).

\section{References}

1. Anastassiades, M.; Scherbaum, E.; Compr. Anal. Chem. 2004, 43, 113 .

2. Anastassiades, M.; Lehotay, S. J.; Stajnbaher, D.; Schenck, F. J.; J. AOAC Int. 2003, 86, 412.

3. Kloepfer, A.; Quintana, J. B.; Reemtsma, T.; J. Chromatogr., A 2005, 1067, 153.

4. Taylor, P. J.; Clin. Biochem. 2005, 38, 328.

5. Manini, P.; Andreoli, R.; Niessen, W. M. A.; J. Chromatogr., A 2004, 1058, 21.

6. Cole, R. B.; Electrospray and MALDI Mass Spectrometry: Fundamentals, Instrumentation, Practicalities and Biological Applications, $2^{\text {th }}$ ed.; Wiley \& Sons: New Jersey, 2010.

7. Cech, N. B.; Enke, C. G.; Anal. Chem. 2000, 72, 2717.

8. Antignac, J. P.; Wasch, K.; Monteau, F.; De Brabander, H.; Andre, F.; Le Bizec, B.; Anal. Chim. Acta 2005, 529, 129.

9. Kruve, A.; Künnapas, A.; Herodes, K.; Leito, I.; J. Chromatogr., A 2008, 1187, 58.

10. Hernández, F.; Sancho, J.; Pozo, O.; Anal. Bioanal. Chem. 2005, $382,934$.

11. Hewavitharana, A. K.; J. Chromatogr., A 2011, 1218, 359.

12. Smeraglia, J.; Baldrey, S. F.; Watson, D.; Chromatographia 2002, 55, S95.

13. Ahumada, D.A.; Zamudio, A.; Rev. Colomb. Quim. 2010, 40, 227.

14. Cech, N. B.; Krone, J. R.; Enke, C. G.; Anal. Chem. 2000, 73, 208.

15. Ferrer, C.; Agüera, A.; Mezcua, M.; Fernández-Alba, A. R.; Mack, D.; Anastassiades, M.; Gamón, M.; J. AOAC Int. 2010, 93, 380 .

16. Cech, N. B.; Enke, C. G.; Mass Spectrom. Rev. 2001, 20, 362. 